\title{
Erratum to: Standardization of a bottle assay-an indigenous method for laboratory and field monitoring of insecticide resistance and comparison with WHO adult susceptibility test
}

\author{
N. Elamathi • Tapan Kumar Barik • Vaishali Verma • \\ Poonam Sharma Velamuri • R. M. Bhatt • S. K. Sharma • \\ Kamaraju Raghavendra
}

Published online: 2 September 2014

(C) Springer-Verlag Berlin Heidelberg 2014

Erratum to: Parasitology Research

DOI 10.1007/s00436-014-4054-y

The original version of this article contained a mistake on the equation and it should read:

$\%$ mortality $=\frac{\text { Number of dead mosquitoes } \times 100}{\text { Number of mosquitoes exposed }}$

The online version of the original article can be found at http:// dx.doi.org/10.1007/s00436-014-4054-y.

N. Elamathi $\cdot$ T. K. Barik $\cdot$ V. Verma $\cdot$ P. S. Velamuri $\cdot$

S. K. Sharma $\cdot$ K. Raghavendra $(\bowtie)$

Vector Control Division, National Institute of Malaria Research

(ICMR), Dwarka Sector 8, New Delhi 110077, India

e-mail: kamarajur2000@yahoo.com

R. M. Bhatt

National Institute of Malaria Research Field Unit, RLTRI Campus,

Lalpur, Raipur 492015, Chhattisgarh, India

Present Address:

T. K. Barik

P.G. Department of Zoology, Berhampur University,

Berhampur 760007, Odisha, India 